\title{
Deep Vein Thrombosis: An Independent Poor Prognosis Factor of Advanced Pancreatic Adenocarcinoma
}

\author{
MARC LAMBERT ${ }^{1}$, ANNE PLOQUIN $^{2}$, LYDIE DECLERCK $^{2}$, ALAIN DUHAMEL $^{3}$, \\ SAMIRA MAKHLOUFI $^{2}$, ANTHONY TURPIN ${ }^{2}$, STÉPHANIE TRUANT ${ }^{4}$ and MOHAMED HEBBAR ${ }^{2}$ \\ Departments of ${ }^{1}$ Internal Medicine and ${ }^{2}$ Medical Oncology, University Hospital, Lille, France; \\ ${ }^{3}$ CERIM, EA2694, UDSL, Department of Biostatistics, University Hospital, Lille, France; \\ ${ }^{4}$ Service Department of Digestive Surgery and Transplantation, University Hospital, Lille, France
}

\begin{abstract}
Background/Aim: Pancreatic adenocarcinoma $(P A)$ is frequently associated with venous thromboembolic events (VTES). Although the prognostic value of VTEs remains controversial, these events can darken the prognosis. In contrast, because they necessitate anticoagulant therapy, some authors found that VTEs improved the prognosis. Indeed, anticoagulants could have an anti-tumor action. Therefore, we evaluated the prognostic value of VTEs in patients with locally advanced or metastatic PA. Patients and Methods: This retrospective study included all patients followed in a medical oncology Department. The prognostic value of tumor parameters, initial patients' characteristics and VTEs were subjected to univariate and multivariate analyses. Results: Based on 142 patients, analyses revealed independent pejorative prognostic value of: VTEs (hazard ratio $(H R)=1.49(95 \%$ confidence interval $(C I)=1.03-2.15) ; p=0.03)$, interval between PA diagnosis and metastases occurrence (HR=0.97 (95\% CI=0.94-0.99), $p=0.02)$ and $\geq 1$ metastatic sites (HR=1.82 (95\% CI $=1.076$ 3.087), $p=0.02$ ). Conclusion: VTEs are an independent factor of poor prognosis in patients with advanced $P A$.
\end{abstract}

Pancreatic adenocarcinoma is one of the most common digestive tract cancers. Its prognosis is highly pejorative with median survival at 6-12 months (1), probably because of its late discovery, usually at a locally advanced or metastatic stage. The parameters linked to cancer could also have a poor prognosis value, especially paraneoplastic thromboses that are

Correspondence to: Prof. Mohamed Hebbar, Service d'Oncologie Médicale, CHRU Hôpital Claude-Huriez, rue Michel-Polonovski, 59037 Lille Cedex, France. Tel: +33 320445461, Fax: +33 320445023,e-mail: mohamed.hebbar@chru-lille.fr

Key Words: Pancreatic adenocarcinoma, deep vein thrombosis, metastasis, prognosis. frequent during pancreatic adenocarcinoma, attesting to more evolved and/or biologically active disease $(2,3)$. Pancreatic adenocarcinoma is the cancer most frequently responsible for thromboembolic events (17-57\%) (4). In addition, anti-cancer treatments increase the thrombotic risk (5). The results of some studies suggested that the occurrence of thromboses aggravated the prognosis (4), perhaps explained by embolic complications of the thromboses themselves, but also because thromboses complicate more advanced and/or aggressive cancers. On the other hand, thromboses require anticoagulant therapy and some observations suggested that these drugs might have an anti-tumor effect. In particular, heparin has been accorded an intrinsic anti-tumor effect, independent of associated treatments (6).

The main objective of this study was to evaluate the prognostic value of venous thromboembolic events (VTEs) occurring in patients with advanced pancreatic cancer.

\section{Patients and Methods}

Patients. This retrospective study included all the patients with locally advanced or metastatic pancreatic adenocarcinoma followed in our Hospital's Department of Medical Oncology between 2001 and 2011. Inclusion criteria were: advanced or metastatic pancreatic adenocarcinoma, histologically proven or satisfying biological and imaging criteria (7); age $>18$ years; World Health Organization performance status (WHO PS) at diagnosis: 0-3; life expectancy estimated at $\geq 2$ months. For this retrospective non-interventional study, written patient consent was not required by French law; however, the Ethics Committee of the CHRU of Lille was informed and gave its approval.

Patients were seen regularly at consultations or during hospitalization and had clinical, laboratory and imaging examinations at regular intervals to evaluate their pancreatic adenocarcinoma and its response to treatment(s). Computedtomography scans were obtained every 2 months to assess tumor progression. This monitoring began at diagnosis and ended at the time of death when the decision to pursue palliative therapy was made or if the patient was lost to follow-up. During physical examinations, venous and arterial thromboses were systematically 
Table I. Comparisons of patients with locally advanced or metastatic pancreatic adenocarcinoma at diagnosis, according to their venous thromboembolic events (VTEs) status.

\begin{tabular}{lccc}
\hline Characteristic & $\begin{array}{c}\text { VTE } \\
(\mathrm{n}=60)\end{array}$ & $\begin{array}{c}\text { No VTE } \\
(\mathrm{n}=82)\end{array}$ & $p$-Value \\
\hline Men/women, n (\%) & $35(58.3 \%) / 25(41.7 \%)$ & $52(63.4 \%) / 30(36.6 \%)$ & 0.53 \\
Age, years, mean (range) & $60.0(41-83)$ & $60.9(28-89)$ & - \\
WHO PS & $5(8.3 \%)$ & $9(11 \%)$ & 0.61 \\
$\quad 0$ & $17(28.3 \%)$ & $21(25.6 \%)$ & 0.70 \\
1 & $26(43.3 \%)$ & $38(46.3 \%)$ & 0.72 \\
2 & $12(20 \%)$ & $14(17.1 \%)$ & 0.65 \\
3 & $14(23.3 \%)$ & $26(31.7 \%)$ & 0.27 \\
Surgery & $58(96.7 \%)$ & $78(95.1 \%)$ & 0.65 \\
Chemotherapy & $29 / 42$ & $4 / 52$ & \\
CA19.9>18,301 IU/mi* & $22(36.7 \%)$ & $32(39.0 \%)$ & 0.0001 \\
$>1$ metastatic site & & \\
\hline
\end{tabular}

*48 missing data: 18/60 patients with DVT and 30/82 patients without DVT. WHO PS, World Health Organization performance status; CA, cancer antigen; DVT, deep vein thrombosis.

sought: questioning the patient; physical examination; and, at the slightest possibility, venous Doppler ultrasonography, pulmonary angio-computed tomography or ventilation-perfusion lung scintigraphy was obtained.

When VTEs occurred, as recommended (8), treatment with lowmolecular-weight heparin or unfractionated heparin was started for at least 3 months.

Statistical analyses. Values of quantitative variables are expressed as means \pm standard deviation (SD) or median (95\% confidence interval (CI)), while those of qualitative parameters are expressed as n (\%). Overall survival curves were obtained with the KaplanMeier method. Survival was defined as the interval between pancreatic adenocarcinoma diagnosis and death. Living patients were censored at the date of the last consultation. All computations were made with SAS software v9.3 (SAS Institute Inc., Cary, NC, USA).

The prognostic value of each qualitative variable was estimated with a log-rank test, while that of each continuous parameter was determined with a Cox model (9). The prognostic value of a VTE was adjusted in a Cox multivariate analysis to the following factors: sex, age, synchronous or metachronous metastasis, metastatic site(s), number of those sites, chemotherapy and/or pulmonary embolism. All those variables were selected à priori based on clinical criteria. Anticoagulant use, which was logically clinically associated with VTEs, was excluded to avoid a colinearity effect. The step-by-step backward-regression model selected variables with $p \leq 0.2$ in univariate analyses to reduce their number. The stability of the subset of selected variables was evaluated with the bootstrap method (10).

With the objective of determining the prognostic value of VTE, considering the range of VTEs rates of $17-57 \%$ (mean=40\%) (4) for patients with pancreatic cancer, all stages combined, estimated median survival of 7 months (3) and retaining median survival of 9.5 months for patients without VTEs and 4.5 months for those with VTEs, to obtain an alpha risk of $5 \%$ and $80 \%$ power; 151 patients (91 without VTEs and 60 with VTEs) had to be included to demonstrate a significant difference between them.

\section{Results}

Demographics and thromboembolic events. Between January 2001 and May 2011, 142 patients met inclusion criteria: 87 $(61.3 \%)$ men and $55(38.7 \%)$ women, mean age $60.6 \pm 10.5$ (median=61.0; range $=28-89$ ) years at the time of diagnosis of locally advanced or metastatic pancreatic cancer. At diagnosis, WHO PS was 0 for $9.9 \%, 1$ for $26.8 \%, 2$ for $45.1 \%$ and 3 for $18.3 \%$. Among the 142 patients, 103 $(75.5 \%)$ have synchronous metastases and $39(27.5 \%)$ subsequently developed metastases, with the number of metastatic sites ranging from 1 to 4 . The mean diagnosis-tometastasis interval was $4.2 \pm 1.2$ months, with 54 (30.7) having multiple metastatic sites. Sixty (42.2\%) patients suffered venous thromboembolic event(s); no arterial thrombosis was observed. The characteristics of the 24 patients with pulmonary emboli, always complicated with deep vein thrombosis (DVT), and 36 with isolated DVT were comparable. The main characteristics of the study population, according to VTE status, are given in Table I.

The mean cancer antigen (CA) 19.9 concentration at diagnosis was $18,301 \pm 5,320 \mathrm{IU} / \mathrm{ml}$. That threshold was exceeded by $29(48.3 \%)$ of the 60 patients with VTE but only $4(4.9 \%)$ of the 82 patients without VTE $(p=0.01)$. Because these values were missing for $48(33.8 \%)$ patients (18/60 patients with VTE and 30/82 patients without VTE), CA19.9 could not be retained for the multivariate analysis. Among the 142 patients studied, 135 (95.1\%) received chemotherapy; because of their poor general condition (WHO PS $\geq 2$ ) or contraindicated hepatic and/or renal insufficiency, the other $7(4.9 \%)$ did not. Forty $(28.2 \%)$ patients underwent surgery for their initially localized disease that subsequently metastasized. 
Table II. Parameters associated with prognosis of pancreatic adenocarcinoma patients according to univariate and multivariate analyses.

\begin{tabular}{lccc}
\hline Parameter & $\begin{array}{c}\text { Univariate } \\
(P)\end{array}$ & $\begin{array}{c}\text { Multivariate } \\
(P)\end{array}$ & $\begin{array}{c}\text { Hazard ratio } \\
(95 \% \text { CI })\end{array}$ \\
\hline Gender & 0.75 & - & - \\
CA19.9 & 0.85 & - & - \\
CA19.9 threshold & 0.14 & - & - \\
Chemotherapy & 0.78 & 0.03 & $1.49(1.03-2.15)$ \\
Pulmonary embolus & 0.27 & 0.02 & $0.97(0.94-0.99)$ \\
Age & 0.95 & 0.02 & $1.82(1.076-3.087)$ \\
Deep vein thrombosis & 0.02 & 0.08 & \\
Diagnosis-to-metastasis interval & 0.08 & & \\
$\geq 1$ metastases & & & \\
\hline
\end{tabular}

CA, Cancer antigen; CI, confidence interval.

Prognosis. Median overall survival was 7.4 (95\% CI=6.59.4) months for the entire population. According to our univariate analysis, median survival was significantly shorter for patients with VTEs (6.5 vs. 8.8 months; $p=0.02$ ) (Figure 1). Only VTE had prognostic value in terms of survival according to that analysis (Table II).

The Cox model included the 3 parameters with $p<0.2$ in the univariate analysis: VTE, diagnosis-to-metastasis interval and number of metastatic sites. The multivariate analysis retained all of them as being independent predictors associated with survival.

\section{Discussion}

This retrospective study included 142 non-selected consecutive patients with locally advanced or metastatic pancreatic adenocarcinoma at diagnosis; $42.2 \%$ of them experienced DVTs. Multivariate analysis of their characteristics retained VTEs, post-diagnosis cancer dissemination and $\geq 1$ metastatic site as being independently associated with shorter survival. Their demographic characteristics at diagnosis were comparable to those previously reported (11).

Khorona et al. described $17 \%-57 \%$ of pancreatic cancer patients as having thrombotic events (4), which is in agreement with our findings. Two studies $(4,12)$ previously showed that VTE in the context of pancreatic cancer was associated with advanced disease and diminished survival, as corroborated by our results of significantly shorter overall survival for patients with VTE (6.5 vs. 8.8 months). The latter might be explained by their more frequent poor general condition (WHO PS 3: $20 \%$ vs. 17.1\%). In addition, keeping in mind that $48(33.8 \%)$ values were missing, patient's CA19.9 concentration was significantly higher for VTE patients, suggesting more advanced disease. A Californian registry-based study on 235,149 cancers showed that the majority of thromboses occurred soon after the diagnosis and

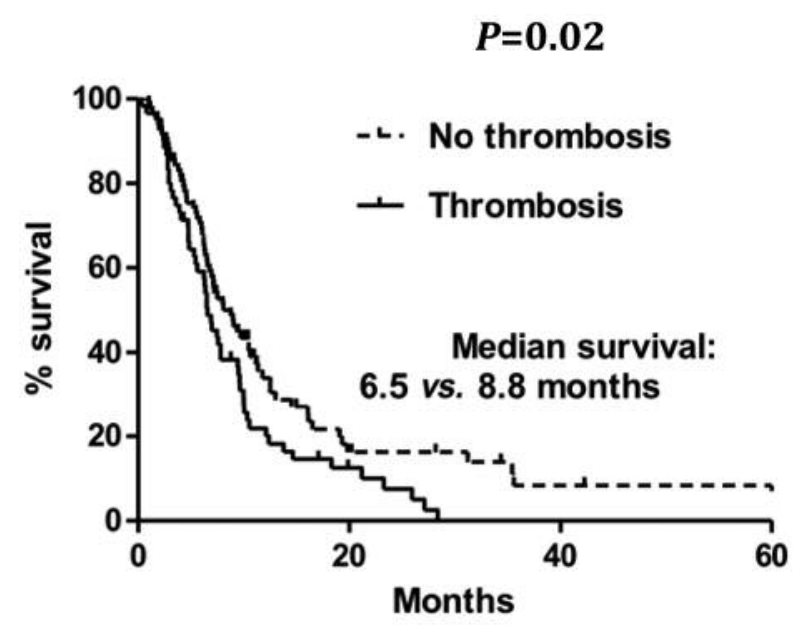

Figure 1. Overall survival of patients with venous thromboembolic events (VTEs) $(n=60)$ or without VTEs $(n=82)$.

that the metastatic stage was the primary risk factor for thrombosis (3). The authors of that study concluded that the occurrence of thrombotic events during the first year after cancer diagnosis was a marker of shorter survival (range of hazard ratios=1.6-4.2; $p<0.1)$. Similarly, another investigation on 227 patients with inoperable pancreatic adenocarcinoma found that a thromboembolic event, concurrent with the diagnosis or during chemotherapy, was associated with a poorer response to treatment and shorter survival (12). According to those investigators, the patients with thromboses had biologically more aggressive cancers than those without. Our results support that hypothesis as we observed no deaths directly attributable to VTEs.

Other researchers reported prolonged survival of patients treated with combined anticoagulants and chemotherapy (13, 14). They hypothesized that the benefit in terms of survival would reflect the immunomodulating or anti-tumor effect of 
heparin, which would facilitate the intracellular penetrations of the chemotherapy molecules. Hence, indirectly, thrombosis during cancer would not be a negative prognosis factor. Indeed, the authors of the PROSPECT-CONKO 004 trial, which compared the adjunction of enoxaparin (a lowmolecular-weight heparin) to the chemotherapy regimen for locally advanced or metastatic pancreatic cancers, concluded that the combination of heparin would not only prevent the thrombotic risk, but also lower the remission rate and prolong survival (13). They also noted that benefit was more marked for patients with localized disease. In addition, improved survival was observed for patients with locally advanced or metastatic pancreatic cancer treated with nadroparin combined with gemcitabine/cisplatin, compared to those receiving chemotherapy alone (14).

The results of our retrospective study covering 10 years confirmed the shorter survival of pancreatic adenocarcinoma patients with VTEs. It is true that only 142 patients, rather than the desired 151, were included but they enabled us to analyze 60 DVTs and, thus, achieve the $80 \%$ power required to demonstrate a significant difference. Survival was not affected by age, sex, chemotherapy, anticoagulation or pulmonary emboli in univariate or multivariate analyses.

A VTE occurred significantly more frequently in patients with advanced pancreatic adenocarcinoma, as shown by the higher number of metastatic sites in our patients with DVTs.

\section{Conclusion}

Our findings showed that VTEs carried an independent pejorative prognostic value for patients with pancreatic adenocarcinoma. The shortened survival observed seems to be linked to the more aggressive phenotype of the cancers rather than those thromboembolic complications.

\section{Conflicts of Interest}

The Authors declare that they have no competing interests.

\section{References}

1 Conroy T, Desseigne F, Ychou M, Bouché O, Guimbaud R, Bécouarn Y, Adenis A, Raoul JL, Gourgou-Bourgade S, de la Fouchardière C, Bennouna $\mathrm{J}$, Bachet JB, Khemissa-Akouz F, Péré-Vergé D, Delbaldo C, Assenat E, Chauffert B, Michel P, Montoto-Grillot C, Ducreux M; Groupe Tumeurs Digestives of Unicancer and PRODIGE Intergroup: Folfirinox versus gemcitabine for metastatic pancreatic cancer. N Engl J Med 364: 1817-1825, 2011.

2 Shah MM and Saif MW: Pancreatic cancer and thrombosis. Highlights from the "2010 ASCO Annual Meeting". Chicago, IL, USA. 4-8 June 2010. JOP 11: 331-333, 2010.
3 Chew HK, Wun T, Harvey D, Zhou H and White RH: Incidence of venous thromboembolism and its effect on survival among patients with common cancers. Arch Intern Med 166: 458-464, 2010.

4 Khorana AA and Fine RL: Pancreatic cancer and thromboembolic disease. Lancet Oncol 5: 655-663, 2004.

5 Levine MN, Gent M, Hirsh J, Arnold A, Goodyear MD, Hryniuk $\mathrm{W}$ and De Pauw S: The thrombogenic effect of anticancer drug therapy in women with stage II breast cancer. N Engl J Med 318: 404-407, 1988.

6 Piccioli A, Falanga A and Prandoni P: Anticoagulants and cancer survival. Semin Thromb Hemost 32: 810-813, 2006.

7 Nouts A, Levy P, Voitot $\mathrm{H}$ and Bernades P: Diagnostic value of serum CA19.9 antigen in chronic pancreatitis and pancreatic adenocarcinoma. Gastroenterol Clin Biol 22: 152-159, 1998.

8 Kearon C, Akl EA, Comerota AJ, Prandoni P, Bounameaux H, Goldhaber SZ, Nelson ME, Wells PS, Gould MK, Dentali F, Crowther $M$ and Kahn SR: American College of Chest Physicians. Antithrombotic therapy for VTE disease: Antithrombotic therapy and prevention of thrombosis, 9th edn. American College of Chest Physicians Evidence-Based Clinical Practice Guidelines. Chest 141(2 Suppl): e419S-494S, 2012.

9 Sauerbrei W, Royston P and Binder H: Selection of important variables and determination of functional form for continuous predictors in multivariable model building, Statist Med 26: 5512-5528, 2007.

10 Sauerbrei W: The use of resampling methods to simplify regression models in medical statistics. Appl Stat 48: 313-429, 1999.

11 Warsame R and Grothey A: Treatment options for advanced pancreatic cancer: a review. Expert Rev Anticancer Ther 12: 1327-1336, 2012.

12 Mandala M, Reni M, Cascinu S, Barni S, Floriani I, Cereda S, Berardi R, Mosconi S, Torri V and Labianca R: Venous thromboembolism predicts poor prognosis in irresectable pancreatic cancer patients. Ann Oncol 18: 1660, 2007.

13 Riess H, Pelzer U, Hilbig A, Stieler J, Opitz B, Scholten T, Kauschat-Brüning D, Bramlage P, Dörken B and Oettle H: Rationale and design of PROSPECT-CONKO 004: A prospective, randomized trial of simultaneous pancreatic cancer treatment with enoxaparin and chemotherapy. BMC Cancer 8 : 361, 2008.

14 Icli F, Akbulut H, Utkan G, Yalcin B, Dincol D, Isikdogan A, Demirkazik A, Onur H, Cay F and Büyükcelik A: Low molecular weight heparin (LMWH) increases the efficacy of cisplatinum plus gemcitabine combination in advanced pancreatic cancer. J Surg Oncol 95: 507-512, 2007.

Received August 25, 2016

Revised September 7, 2016

Accepted September 8, 2016 